\title{
Recovery rate and determinants of severe acute malnutrition children treatment in Ethiopia: a systematic review and meta- analysis
}

Kassahun Gebeyehu Yazew ${ }^{1 *}$ D, Chanyalew Worku Kassahun ${ }^{1}$, Amare Wondim Ewnetie', Habtamu Kerebih Mekonen ${ }^{2}$ and Endalamaw Salilew Abagez ${ }^{2}$

\begin{abstract}
Background: Severe acute malnutrition affects more than 20 million children. Africa is pointed out as a region where the problem is highly prevalent. There were individual studies on the recovery rate and its determinants among children with severe acute malnutrition in Ethiopia. But, there is no national pooled estimate. Therefore, this systematic review and meta-analysis aimed to estimate the recovery rate and determinants among children with severe acute malnutrition admitted to the therapeutic feeding unit in Ethiopia.

Methods: The Preferred Reporting Items for Systematic Reviews and Meta-Analyses guideline was followed in this study. Studies were accessed through electronic web-based search from PubMed, Cochrane Library, Google Scholar, and EMBASE. The statistical analysis was conducted using STATA version-11 software. The pooled prevalence was estimated with 95\% confidence intervals using a random-effects model.

Result: A total of 12 studies were included with 2658 participants in the analysis. The overall pooled estimated recovery rate among children with severe acute malnutrition admitted to the inpatient therapeutic feeding unit in Ethiopia was $72.02 \%(\mathrm{Cl}, 64.83,79.22 \%)$. In the subgroup analysis, the highest estimate (80.29\%) was observed in studies conducted in Oromia regional state, while $68.63 \%$ was observed in studies Southern Nation Nationality of people region $68.63 \%$. Children who had no congestive heart failure were 4.88 times $(\mathrm{OR}, 4.88 ; 95 \% \mathrm{Cl}, 2.246$, 10.586) more likely to recover than their counterparts.
\end{abstract}

Conclusion: The recovery rate among severe acute malnourished children on the therapeutic feeding unit in Ethiopia lied within the international minimum sphere. Hence, health care providers shall strengthen the management of severe acute malnutrition and management other co-morbidities like congestive heart failure.

Systematic review registration: PROSPERO CRD42019119124

Keywords: Recovery rate, Therapeutic feeding unit, Severe acute malnutrition, Under-five children, Children, Ethiopia

\footnotetext{
* Correspondence: kassish6@gmail.com

${ }^{1}$ School of Nursing, College of Medicine and Health Science, University of

Gondar, Gondar, Ethiopia

Full list of author information is available at the end of the article
}

(c) The Author(s). 2019 Open Access This article is distributed under the terms of the Creative Commons Attribution 4.0 International License (http://creativecommons.org/licenses/by/4.0/), which permits unrestricted use, distribution, and reproduction in any medium, provided you give appropriate credit to the original author(s) and the source, provide a link to the Creative Commons license, and indicate if changes were made. The Creative Commons Public Domain Dedication waiver (http://creativecommons.org/publicdomain/zero/1.0/) applies to the data made available in this article, unless otherwise stated. 


\section{Background}

Severe acute malnutrition (SAM) is stated as a weightfor-height measurement of $70 \%$ or more below the median, or three SD or more below the mean National Center for Health Statistics reference points, which is called "wasted"; the occurrence of two-sided pitting edema of nutritional origin, which is called "oedematous malnutrition"; or a mid-upper-arm circumference of less than $110 \mathrm{~mm}$ in children aged $1-5$ years [1].

Globally, it is valued that there are nearly 20 million children who are severely acutely malnourished. Most of them live in South Asia and Sub-Saharan Africa [2].

Malnutrition can affect all age groups but is more frequent among infants and young children (4-6 years). Malnutrition contributes to $50-60 \%$ of the child deaths for which infection is the underlying cause [3, 4]. A literature review conducted in Africa showed that children with SAM given RUTF were 51\% more likely to achieve nutritional recovery than the standard care group [5].

In developing countries, $2 \%$ of children suffer from severe acute malnutrition [6]. In India, $2.8 \%$ of children under five are severely wasted [7]. The United Nations Children's Fund estimated sixty thousand children to be severely malnourished in Ethiopia [8]. In children younger than 5 years of age, according to the Ethiopian Demographic and Health Survey (EDHS) report, 11\% are wasted, 2\% severely wasted, $38 \%$ underweight (below - 2 SD), and 16\% severely underweight (below - 3 SD) [9]. Among the principal causes of death in young children, $60.7 \%$ of deaths from diarrhea, $52.3 \%$ of deaths from pneumonia, $44.8 \%$ of deaths from measles, and $57.3 \%$ of deaths from malaria are attributable to under-nutrition [10]. According to the United Nations International Emergency Fund (UNICEF) estimates, around 26 million under-five children suffer from SAM in developing countries [11].

Despite the huge effects of the recovery rate in the treatment of children with severe acute malnutrition and its importance as a public health problem in Ethiopia, the overall recovery rate among children treated with severe acute malnutrition in the country level remains unknown. Therefore, the objective of this systematic review and meta-analysis is to estimate the pooled recovery rate and its determinants among children with severe acute malnutrition admitted to the inpatient therapeutic feeding unit in Ethiopia.

\section{Materials and methods Study protocol registration} PROSPERO database with protocol number CRD42019119124.

\section{Search strategy}

We made an inclusive literature search conducted from October 2018 to January 2018 from PubMed, Cochrane
Library, Google Scholar, CINAHL, and EMBASE. A selection of publications, data extraction, and reported results for the review was designed in accordance with the Preferred Reporting Items for Systematic Reviews and Meta-Analyses (PRISMA) guidelines [12]. Throughout the comprehensive literature search, the following search terms were used: "Recovery Rate and Determinants of the Treated Severe Acute Malnutrition Children in Ethiopia," "Recovery rate OR treatment outcome in Ethiopia," and "Recovery rate AND children in Ethiopia." Furthermore, we checked the reference lists of published studies to identify additional articles.

\section{Selection and identification of studies}

A total of 2658 studies were identified from the literature search. We added one gray literature that was not found in the search. Of these studies, 9 articles of duplicate records were identified and removed. A total of 2625 articles were excluded after reviewing the titles and abstracts (because 2581articles were irrelevant and 19 articles were done at Health Center). After assessing the full texts of the remaining articles, 13 additional articles were excluded because of the following: 6 articles are of poor quality, 5 articles do not report outcome interest, and 2 articles were done outside Ethiopia. Therefore, a total of twelve unique studies were eligible and enrolled for final analysis (Fig. 1).

\section{Study selection and eligibility criteria}

We included all studies that were conducted on the recovery rate and determinants of severe acute malnutrition children treatment. The participants were SAM children with age between 0 and180 months, regardless of their sex and other characteristics. We included all article types that were published in the form of journal articles, master's thesis, and dissertations in English. Moreover, studies which failed to report recovery rate and those studies conducted on adult were excluded. No restriction was made to the date of publication that was conducted only in Ethiopia.

\section{Outcome measures \\ Research questions}

1. What is the estimated pooled prevalence of the recovery rate among children with severe acute malnutrition admitted to the inpatient therapeutic feeding unit?

2. What are the determinants that affect the recovery rate among children with severe acute malnutrition admitted to the inpatient therapeutic feeding unit?

The primary outcome of this systematic review and meta-analysis was measuring the prevalence of the 


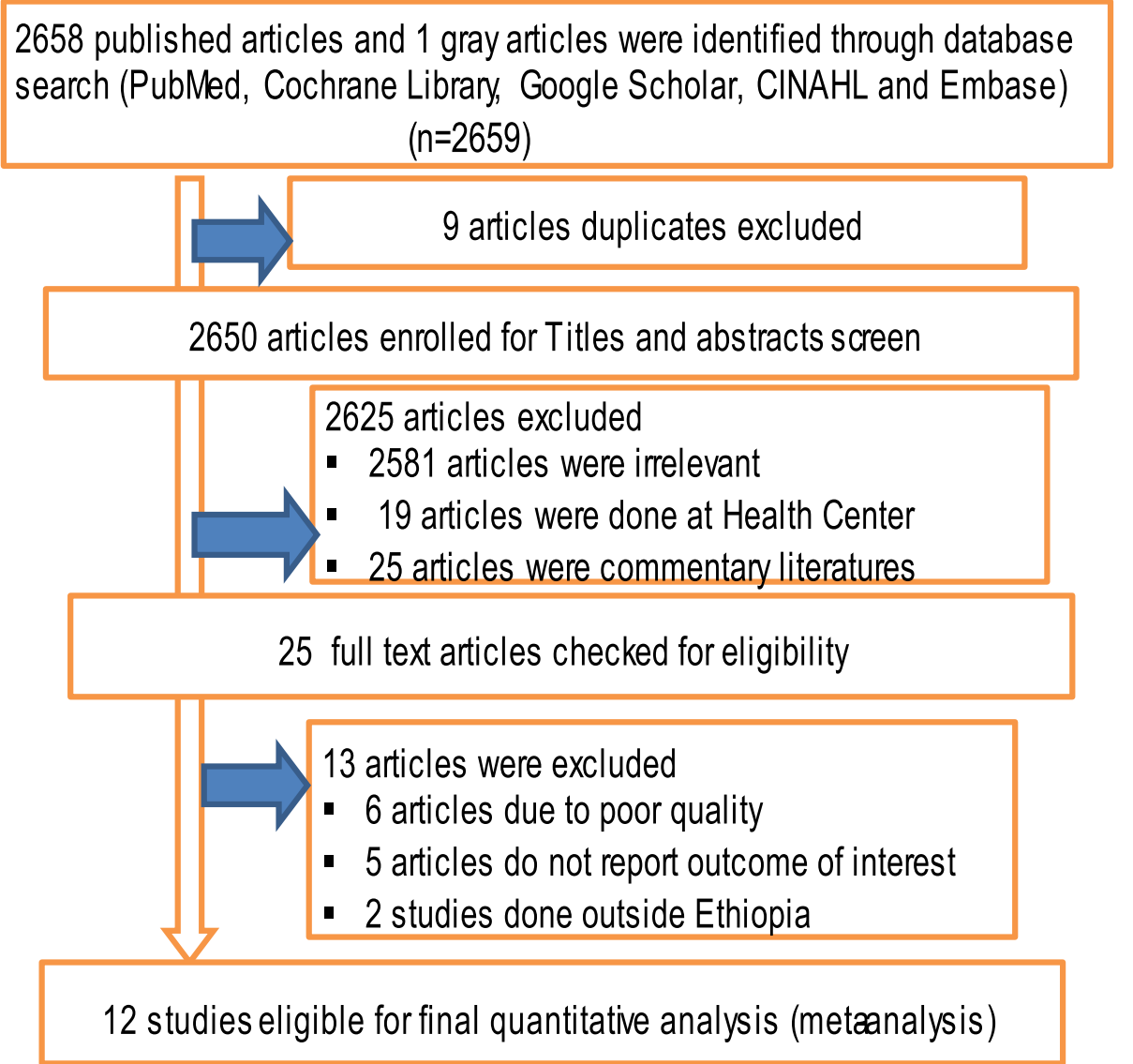

Fig. 1 Flow chart of study selection for meta-analysis of recovery rate of the treated severe acute malnutrition children in Ethiopia, 2018

recovery rate among children with severe acute malnutrition admitted to the inpatient therapeutic feeding unit presented as a percentage of the overall participants.

The secondary outcome was assessed determinants influencing the recovery rate among children with severe acute malnutrition admitted to the inpatient therapeutic feeding unit.

\section{Quality assessment and critical appraisal}

The qualities of each article were assessed by using a critical appraisal tool (JBI) for use in systematic reviews of the prevalence study [13]. Those studies less than 50\% of the quality assessment checklist criteria were excluded. The methodological quality of the identified articles was also assessed by two reviewers (K.G. and C.W.) independently, and disagreements among reviewers were fixed accordingly through a discussion with a third reviewer $(\mathrm{A} . \mathrm{W})$ when necessary

\section{Data extraction}

Data were extracted using pre-piloted data extraction forms which were developed by the two authors (E.S. and H.K). It included names of author, year of publication, interventions, durations, the region in the country, study design, sample size, number of people with the outcome, and overall prevalence.

\section{Data analysis and synthesis}

The extracted data in a Microsoft Excel spreadsheet were imported to STATA v. 11 for analysis. The analysis was done by the authors using STATA. A randomeffects model was used to estimate the overall pooled magnitude. The DerSimonian and Laird method, which assumes heterogeneity across studies, is the most common method for using a random-effects model in the meta-analysis $[14,15]$. A random-effects meta-analysis is also recommended for use when heterogeneity between studies exists [16]. The heterogeneity of articles was tested using ${ }^{2}$ test statistics, that ranged from 0 to $100 \%$. A value of $0 \%$ indicates no observed heterogeneity, while $100 \%$ indicates significant heterogeneity. A $p$ value of less than 0.05 was used to declare heterogeneity [17]. In this meta-analysis, $I^{2}$ values were found to be high (> 75\%). Moreover, the presence of heterogeneity was also assessed by subgroup analysis and metaregression. A visual inspection of the publication bias 
was conducted using a funnel plot. An asymmetry of the funnel plot is an indicator of publication bias [18]. Eggers and Begg's tests were also conducted to check the potential publication bias. A $p$ value of less than 0.05 was used to declare the statistical significance of publication bias [19]. Additionally, the sensitivity analysis was also done to assess whether the pooled prevalence estimates were affected by single studies.

\section{Results}

\section{Characteristics of included studies}

A total of 12 studies with 4890 participants were included in this meta-analysis and are summarized in Table 1. The studies were conducted from 2008 to 2018 in different regions of the country. Among 12 studies, five of them $[22,26-28,30]$ were in the Amhara region, two studies [24, 31] were in SNNP, four studies [20, 21, $23,25]$ were in the Oromia region, and one study [29] was from the Dredewa region. A study in both minimum [25] and maximum [20] sample size was conducted in the Oromia region. In terms of study design, 3 studies $[21,22,28]$ were conducted by cross-sectional, 7 studies $[22,23,25-298,31]$ were conducted by retrospective cohort, and two studies $[23,25]$ were conducted by retrospective case-control (Table 1).

\section{The recovery rate of the treatment of SAM children} (meta-analysis)

The estimated pooled recovery rate of the treatment of SAM children reported by the 12 studies was 72.02 (95\%
CI, 64.83, 79.22\%) with significant heterogeneity between studies $\left(I^{2}=97.2 \%, p \leq 0.001\right)$ (Fig. 2).

In the subgroup analysis by the study area and study designs, the highest estimated recovery rates of $80.29 \%$, $95 \%$ CI $72.27,88.32, I^{2}=94.5 \%$ and $68.63 \%, 95 \%$ CI $50.50,86.76 \%, I^{2}=96.2 \%$ were found in studies conducted in Oromia and SNNP regional states respectively (Additional file 1: Figure S1). Case-control studies account the highest estimate $(88.06 \%, 95 \%$ CI 85.6, 90.52, $I^{2}=0.0 \%$ ) (Additional file 2: Figure S2).

\section{Investigation of heterogeneity}

Heterogeneity in systematic reviews and meta-analysis results of studies are inevitable due to the difference in study quality, methodology, sample size, and inclusion criteria for participants [32, 33]. In this meta-analysis, the value of $I^{2}$ is definitely an indication of significantly high heterogeneity, so we conducted the analysis with a random-effects model to adjust for the observed variability. Furthermore, the presence of heterogeneity was also assessed by subgroup analysis (study area and study designs). However, the level of heterogeneity was high after the subgroup analysis discussed above (Additional file 1: Figure S1 and Additional file 2: Figure S2). Then, we further try to investigate the source of heterogeneity using a meta-regression model with publication year and sample size as covariates. Meta-regression is a more complex and preferable method than subgroup analysis for investigating heterogeneity and has the potential advantage of allowing the assessment of one or more covariates simultaneously [34]. The result of the meta-regression

Table 1 Characteristics of studies included in meta-analysis of SAM recovery rate in Ethiopia, 2018

\begin{tabular}{|c|c|c|c|c|c|c|c|c|c|c|}
\hline $\begin{array}{l}\text { S. } \\
\text { no }\end{array}$ & Author/s (reference) & $\begin{array}{l}\text { Publication } \\
\text { year }\end{array}$ & Interventions & $\begin{array}{l}\text { Durations in } \\
\text { days }\end{array}$ & $\begin{array}{l}\text { Age in } \\
\text { months }\end{array}$ & Region & $\begin{array}{l}\text { Study } \\
\text { design }\end{array}$ & $\begin{array}{l}\text { Sample } \\
\text { size }\end{array}$ & Case & $\begin{array}{l}\text { Prevalence\% } \\
(95 \% \mathrm{Cl})\end{array}$ \\
\hline 1 & Jarso et al. [20] & 2015 & TF & 17.4 & $6-59$ & Ormia & Cohort & 947 & 737 & $77.8(75.15,80.45)$ \\
\hline 2 & MB Mena et al. [21] & 2018 & $\mathrm{TF}+\mathrm{M}$ & 21 & $0-180$ & Ormia & CS & 205 & 137 & $66.8(60.35,73.25)$ \\
\hline 3 & Abeje AT, et al. [22] & 2016 & TF & 35 & $2-59$ & Amhr & CS & 298 & 204 & $68.5(63.23,73.77)$ \\
\hline 4 & A. Berti et al. [23] & 2008 & TF & 12.5 & $0-59$ & Orm & CC & 493 & 436 & $88.4(85.57,91.23)$ \\
\hline 5 & Kabeta A et al. [24] & 2017 & $\mathrm{TF}+\mathrm{M}$ & 18.16 & $0-59$ & SNNP & Cohort & 196 & 153 & $78(72.20,83.80)$ \\
\hline 6 & $\begin{array}{l}\text { Chalachew M et al. } \\
\text { [25] }\end{array}$ & 2014 & TF & 21 & $0-168$ & Ormia & $\mathrm{CC}$ & 173 & 150 & $87(74.34,83.26)$ \\
\hline 7 & Mekuria et al. [26] & 2017 & $\mathrm{TF}+\mathrm{M}$ & 11 & $6-59$ & Amhra & Cohort & 253 & 197 & $77.9(72.79,83.01)$ \\
\hline 8 & T Chane et al. [27] & 2014 & $\mathrm{TF}+\mathrm{M}$ & 14 & $0-59$ & Amhara & Cohort & 324 & 275 & $85(81.11,88.89)$ \\
\hline 9 & $\begin{array}{l}\text { Desyibelew HD et al. } \\
\text { [28] }\end{array}$ & 2017 & $\mathrm{TF}+\mathrm{M}$ & 23 & $6-59$ & Amhara & CS & 401 & 234 & $58.4(53.58,63.22)$ \\
\hline 10 & Oumer et al. [29] & 2016 & $\mathrm{TF}+\mathrm{M}$ & 9 & $0-59$ & Diriedewa & Cohort & 617 & 431 & $69.9(66.28,73.52)$ \\
\hline 11 & Desta et al. [30] & 2015 & TF & 32 & $0-59$ & Amhara & Cohort & 415 & 193 & $46.5(41.70,51.30)$ \\
\hline 12 & $\begin{array}{l}\text { Tadele Girum et al. } \\
\text { [31] }\end{array}$ & 2017 & $T F+M$ & 14 & $0-59$ & SNNP & Cohort & 568 & 338 & $59.5(55.46,63.54)$ \\
\hline
\end{tabular}

CC case-control, CS cross-sectional, M medications, TF therapeutic foods, SNNP Southern Nation and Nationalities of people Therapeutic foods include F75, F100 and plump net

Medications are drugs given during admission with therapeutic foods (Amoxicillin, Ampicillin, Gentamycin, Vitamin A, Folic acid, Albendazole/Mebendazole) 


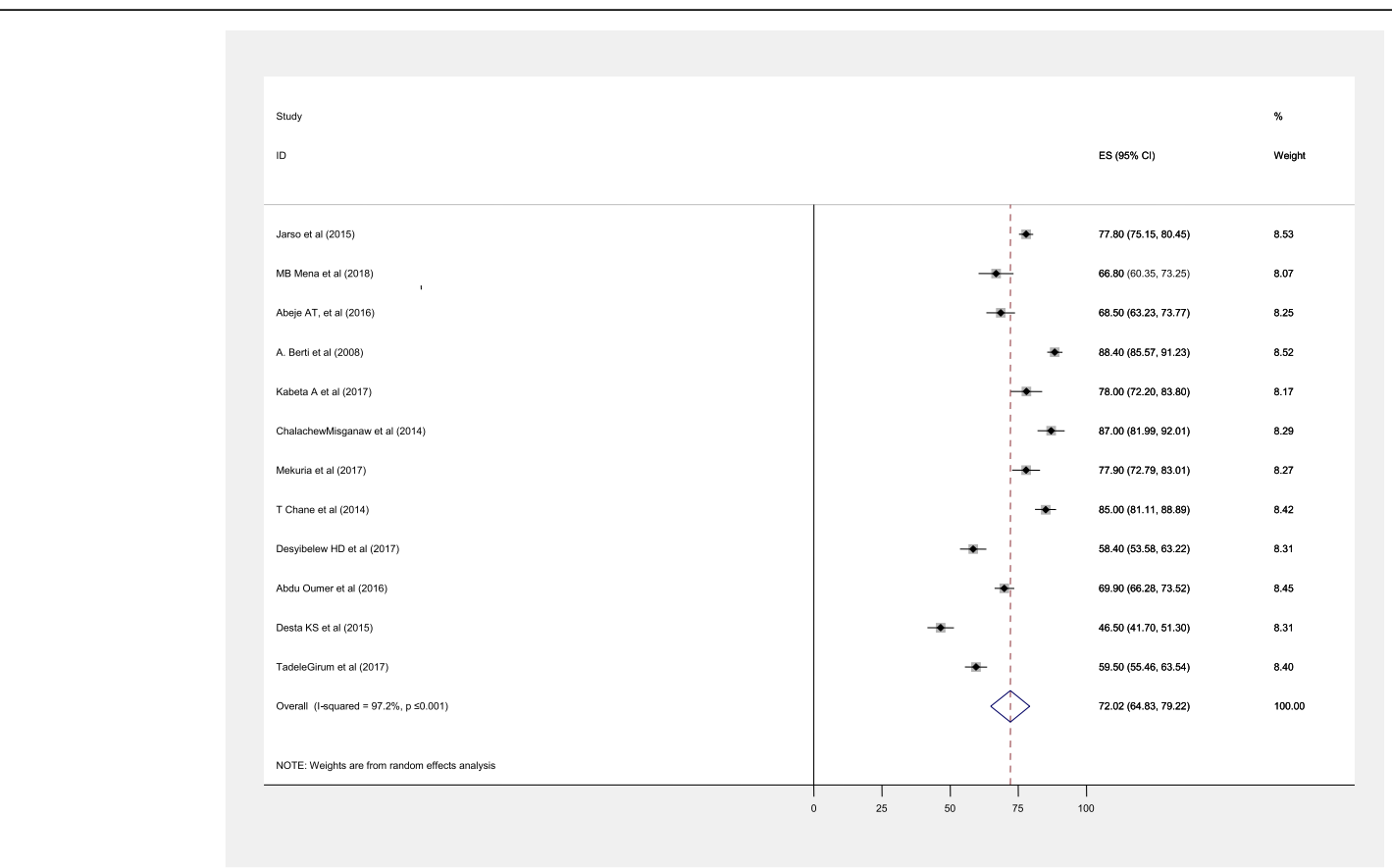

Fig. 2 Forest plot showing the pooled recovery rate of the treated SAM children in Ethiopia, 2018

analysis showed that both covariates were not statistically significant for the presence of the heterogeneity (Additional file 3: Table S1).

\section{Publication bias}

The presence of publication bias was examined using funnel plots and tests (Egger's and begs). Each point in funnel plots represents no separate study, and symmetrical distribution is evidence of the absence of publication bias [35]. First, each study's effect size was not plotted against the standard error and visual inspection of the funnel plot suggests asymmetry, as 3 studies lay on the right side and nine studies on midline representing the pooled prevalence (Additional file 4: Figure S3). We also performed Egger's, and Bag's tests to investigate the publication bias. The result of these tests was not showing the significant evidence of the publication bias ( $p$ value $>0.05$ ) (Additional file 5: Table S2).

\section{Sensitivity analysis}

The result indicated that no single study unduly influenced the overall estimate of the recovery rate among children with SAM on treatment (Additional file 6: Figure S4).

\section{Factors assessed}

Seven studies [21, 22, 24, 27-30] were included in the analysis of determinant factors of the recovery rate. Greater heterogeneity was observed among studies. Five factors were assessed in the quantitative meta-analysis. Six articles for the presence of HIV/AIDS [21, 22, 27-30] with
2828 participants, two articles for the presence of CHF $[27,28]$ with 725 participants, three articles for the presence of anemia [21, 24, 28] with 802 participants, and five articles for presence of TB [24, 27-30] with 1953 participants were assessed for their associations to the recovery rate of treated SAM children.

Of the factors, the presence of CHF was the significant factors of the recovery rate. Children who had no congestive heart failure were 4.88 times (OR 4.88, 95\% CI $2.246,10.586)$ more likely to recover than their counterparts (Additional file 7: Figure S5).

\section{Discussion}

Despite the effort implemented to reduce malnutrition, the proportion of severe acute malnutrition treatment recovery rate in the therapeutic feeding unit is still lower. As far as is known, there are no previous systematic reviews/ meta-analyses that have examined the national estimate of the recovery rate and its determinants among severe acute malnutrition among children in Ethiopia.

The result of this meta-analysis indicated that the overall recovery rate among admitted children with SAM to therapeutic feeding unit was 72.02 . This finding is in line with the international standard recommendation of the minimum recovery rate is greater than $75 \%$ [36]. It is also similar to findings conducted in 13 African countries 73\% [37] and Kenya 73.3\% [38] studies. This finding is higher than the studies done from different countries; in India 51.7\% [39], Pakistan 50\% [40], and in low and middle-income countries 51\% [5]. The 
difference might be due to differences in study design, study population, socioeconomic status, quality of care provided for children, health-seeking behavior, availability, and the accessibility of therapeutic foods and medications. However, the result of this finding was lower than studies recruited at Bangladesh 92\% [41], Niger 91.4\% [42], Malawi 89\% [43], and Sudan 82\% [44]. The possible justification can be sample size and study setting.

The results of this review highlighted common determinant factors for the recovery rate among children with SAM on the treatment. Even though there is significant heterogeneity in odds ratios, the study had an odds ratio of greater than one which indicates an increased risk for recovery rate among children with SAM on treatment.

As it is indicated in the seven studies, the presence of $\mathrm{CHF}$ was the significant determinant factors of recovery rate among children with SAM on treatment in Ethiopia. The outcome of this finding was supported by the studies conducted in low- and middle-income countries [5] and South Africa [45].

\section{Conclusion}

The proportion of the recovery rate lied on the minimum sphere of the international standard for the treated SAM children. The present of CHF affects the recovery rate of severe acute malnutrition treatment among children in Ethiopia. Hence, health care providers shall strengthen the management of severe acute malnutrition and management other co-morbidities like $\mathrm{CHF}$.

\section{Potential limitations}

Like other systematic review and meta-analysis, this review has some drawbacks. The first drawback of this review was only English articles, or reports were considered to conduct this nationally based review. In addition, some of the studies included in this review were cross-sectional in nature because the outcome variable might be affected by other confounding variables. Hence, this factor could affect the estimated result. Furthermore, this review represented only studies reported from four regions of the country. Therefore, the regions may be under-represented due to the limited number of articles included.

\section{Supplementary information}

Supplementary information accompanies this paper at https://doi.org/10. 1186/s13643-019-1249-4.

Additional file 1: Figure S1. Subgroup analysis by regions on the recovery rate of the treatment among SAM children Ethiopia, 2018

Additional file 2: Figure S3. Subgroup analysis by study designs on treatment recovery rate among SAM children Ethiopia, 2018

Additional file 3: Table S1. Meta-regression analysis of factors with heterogeneity of the recovery rate of children with SAM treatment in Ethiopia, 2018
Additional file 4: Figure S2. Funnel plots to test, the publication bias of the 12 studies, 2018

Additional file 5: Table S2. Publication bias of recovery rate of the treatment among SAM children in Ethiopia, 2018

Additional file 6: Figure S3. Result of Sensitivity analysis of the 12 studies, 2018

Additional file 7: Figure S4. Forest plot depicting the pooled odds ratio (log scale) of the associations between recovery rate and its determinant (Presence of CHF), 2018

Additional file 8. PRISMA checklist

\section{Abbreviations}

CHF: Chronic heart failure; DD: Driedewa; HIV/AIDS: Human immune virus/ acquired immune deficiency syndrome; JBI: Joanna Brigg's Institute; OR: Odds ratio; SAM: Severe acute malnutrition; SNNP: Southern Nation, Nationality of people; TFP: Therapeutic feeding program

\section{Acknowledgements}

Not applicable.

\section{Authors' contributions}

KG involved in the design, selection of articles, data extraction, statistical analysis, and manuscript writing. CW involved in selection of articles, statistical analysis, and manuscript editing. A.W, H.K and E.S are also involved in data extraction, analysis, and manuscript editing. All the authors read and approved the final draft of the manuscript.

\section{Authors' information}

KG is a lecturer and practitioner nurse at the Department of Medical Nursing, School of Nursing, College of Medicine and Health Science, University of Gondar, Gondar, Ethiopia.

$\mathrm{CW}$ is a lecturer and practitioner nurse at the Department of comprehensive Nursing, School of Nursing, College of Medicine and Health Science, University of Gondar, Gondar, Ethiopia.

AW is a lecturer and practitioner nurse at the Department of Pediatric Nursing, School of Nursing, College of Medicine and Health Science, University of Gondar, Gondar, Ethiopia.

HK is assistant professor at the Department of Psychiatric, College of Medicine and Health Science, University of Gondar, Gondar, Ethiopia. ES is a lecturer and practitioner nurse at the Department of Psychiatric, College of Medicine and Health Science, University of Gondar, Gondar, Ethiopia.

\section{Funding}

Not applicable.

Availability of data and materials

Data will be available on the request of the corresponding author.

Ethics approval and consent to participate

Not applicable.

Consent for publication

Not applicable.

Competing interests

The authors declare that they have no competing interests.

\section{Author details}

${ }^{1}$ School of Nursing, College of Medicine and Health Science, University of Gondar, Gondar, Ethiopia. ${ }^{2}$ Department of Psychiatric, College of Medicine and Health Science, University of Gondar, Gondar, Ethiopia.

Received: 8 June 2019 Accepted: 3 December 2019

Published online: 13 December 2019

References

1. Collins S, Dent N, Binns P, Bahwere P, Sadler K, Hallam A. Management of severe acute malnutrition in children. Lancet. 2006;368(9551):1992-2000. 
2. Organization WH. World Food Program, United Nations System Standing Committee on Nutrition, United Nations Children's Fund (2007) Community-based management of severe acute malnutrition. Geneva: World Health Organization; 2008.

3. MoH F. National strategy for child sunvival in Ethiopia. Ethiopia: Addis Ababa; 2005.

4. WHO U. Basics Nutrition Essentials: a guide for health managers. Geneva: World Health organization; 1999.

5. Lenters LM, Wazny K, Webb P, Ahmed T, Bhutta ZA. Treatment of severe and moderate acute malnutrition in low- and middle-income settings: a systematic review, meta-analysis and Delphi process. BMC Public Health. 2013;13(3):S23.

6. Collins S, Sadler K, Dent N, Khara T, Guerrero S, Myatt M, et al. Key issues in the success of community-based management of severe malnutrition. Food Nutr Bull. 2006;27(3_suppl3):S49-82.

7. Bhan MK, Bhandari N, Bahl R. Management of the severely malnourished child: perspective from developing countries. Bmj. 2003;326(7381):146-51.

8. Grobler-Tanner C, Collins S. Community Therapeutic Care (СTC): a new approach to managing acute malnutrition in emergencies and beyond; 2004

9. Balaseltan EYs, Macro O. Ethiopia demographic and health survey, 2005: Central Statistical Authority; 2006.

10. Caulfield LE, de Onis M, Blössner M, Black RE. Undernutrition as an underlying cause of child deaths associated with diarrhea, pneumonia, malaria, and measles. Am J Clin Nutr. 2004;80(1):193-8.

11. Esubalew D, Kiflu M, Tessema T. Community based emergency response to reduce acute malnutrition and improved access to nutritional support and care. Jarcoo consulting. 2009

12. Liberati A, Altman DG, Tetzlaff J, Mulrow C, Gøtzsche PC, loannidis JP, et al. The PRISMA statement for reporting systematic reviews and meta-analyses of studies that evaluate health care interventions: explanation and elaboration. PLoS Med. 2009;6(7):e1000100.

13. Munn Z, Moola S, Riitano D, Lisy K. The development of a critical appraisal tool for use in systematic reviews addressing questions of prevalence. Int $J$ Health Policy Manag. 2014;3(3):123.

14. George BJ, Aban IB. An application of meta-analysis based on DerSimonian and Laird method. Springer; 2016.

15. IntHout J, loannidis JP, Borm GF. The Hartung-Knapp-Sidik-Jonkman method for random effects meta-analysis is straightforward and considerably outperforms the standard DerSimonian-Laird method. BMC Med Res Methodol. 2014:14(1):25.

16. Knol M, Twisk JW, Beekman AT, Heine R, Snoek FJ, Pouwer F. Depression as a risk factor for the onset of type 2 diabetes mellitus. A meta-analysis. Diabetologia. 2006;49(5):837.

17. Park M, Katon WJ, Wolf FM. Depression and risk of mortality in individuals with diabetes: a meta-analysis and systematic review. Gen Hosp Psychiatry. 2013;35(3):217-25.

18. Nouwen A, Winkley K, Twisk J, Lloyd CE, Peyrot M, Ismail K, et al. Type 2 diabetes mellitus as a risk factor for the onset of depression: a systematic review and meta-analysis. Springer; 2010.

19. Sarokhani D, Delpisheh A, Veisani Y, Sarokhani MT, Manesh RE, Sayehmiri K. Prevalence of depression among university students: a systematic review and meta-analysis study. Depress Res Treat. 2013;2013.

20. Jarso H, Workicho A, Alemseged F. Survival status and predictors of mortality in severely malnourished children admitted to Jimma University Specialized Hospital from 2010 to 2012, Jimma, Ethiopia: a retrospective longitudinal study. BMC Pediatr. 2015;15(1):76.

21. Mena MB, Dedefo MG, Billoro BB. Treatment outcome of severe acute malnutrition and its determinants among pediatric patients in West Ethiopia. Int J Pediatr. 2018;2018.

22. Ashagrie Terefe Abeje TWG, Malefia YD, Befftu BB. Analysis of hospital records on treatment outcome of severe acute malnutrition: the case of Gondar University Tertiary Hospital. Pediatric therapeutics. 2016;6(2):1000283.

23. Berti A, Bregani ER, Manenti F, Pizzi C. Outcome of severely malnourished children treated according to UNICEF 2004 guidelines: a one-year experience in a zone hospital in rural Ethiopia. Trans R Soc Trop Med Hyg. 2008:102(9):939-44.

24. Kabeta A, Bekele G. Factors associated with treatment outcomes of under-five children with severe acute malnutrition admitted to therapeutic feeding unit of Yirgalem Hospital. Clinics Mother Child Health. 2017;14(261):2.

25. Misganaw C, Mesfin M, Tesfaye M, Derese A. Retrospective study on outcome of in-patient treatment of severe acute malnutrition in Jimma University specialized hospital from September 2011-September 2012. Journal of Diagnostics. 2014;1(2):18-28.
26. Mekuria G, Derese T, Hailu G. Treatment outcome and associated factors of severe acute malnutrition among 6-59 months old children in Debre Markos and Finote Selam hospitals, Northwest Ethiopia: a retrospective cohort study. BMC Nutrition. 2017:3(1):42.

27. Chane T, Oljira L, Atomesa E, Agedew E. Treatment outcome and associated factors among under-five children with severe acute malnutrition admitted to therapeutic feeding unit in Woldia hospital, North Ethiopia. Nutrition \& Food Sciences. 2014

28. Desyibelew HD, Fekadu A, Woldie H. Recovery rate and associated factors of children age 6 to 59 months admitted with severe acute malnutrition at inpatient unit of Bahir Dar Felege Hiwot Referral hospital therapeutic feeding unite, northwest Ethiopia. PLoS One. 2017;12(2):e0171020.

29. Oumer A, Mesfin F, Demena M. Survival status and predictors of mortality among children aged 0-59 months admitted with severe acute malnutrition in dilchora referral hospital, Eastern Ethiopia. East African Journal of Health and Biomedical Sciences. 2016;1(1):13-22.

30. Desta K. Survival status and predictors of mortality among children aged 059 months with severe acute malnutrition admitted to stabilization center at Sekota Hospital Waghemra Zone. J Nutr Disord Ther. 2015;5:160.

31. Girum T, Kote M, Tariku B, Bekele H. Survival status and predictors of mortality among severely acute malnourished children $<5$ years of age admitted to stabilization centers in Gedeo Zone: a retrospective cohort study. Ther Clin Risk Manag. 2017;13:101.

32. Baker W, Michael White C, Cappelleri J, Kluger J, Coleman C. From the Health Outcomes P, et al. Understanding heterogeneity in meta-analysis: the role of meta-regression. Int J Clin Pract. 2009:63(10):1426-34.

33. Melsen W, Bootsma M, Rovers M, Bonten M. The effects of clinical and statistical heterogeneity on the predictive values of results from metaanalyses. Clin Microbiol Infect. 2014;20(2):123-9.

34. Hardy RJ, Thompson SG. Detecting and describing heterogeneity in metaanalysis. Stat Med. 1998;17(8):841-56.

35. Egger M, Smith GD, Schneider M, Minder C. Bias in meta-analysis detected by a simple, graphical test. Bmj. 1997;315(7109):629-34.

36. Layton R. The Sphere Project: Humanitarian charter and minimum standards in disaster response [book review]. Australian Journal of Emergency Management, The. 2001;16(1):37.

37. Yvonne G. Management of severe acute malnutrition in Africa. University of Aberdeen, Department of Medicine and Therapeutics: UK. 2000;8:24-32.

38. Mbaya D, Bitok LK, Karani AK, Osano B, Habtu M. Outcomes of severely malnourished children aged 6-59 months on outpatient management program in Kitui County Hospital, Kenya. Open Journal of Pediatrics. 2015; 5(04):326.

39. Singh K, Badgaiyan N, Ranjan A, Dixit HO, Kaushik A, Kushwaha KP, et al. Management of children with severe acute malnutrition: experience of Nutrition Rehabilitation Centers in Uttar Pradesh, India. Indian Pediatr. 2014; 51(1):21-5.

40. Ahmed S, Ejaz K, Mehnaz A, Adil F. Implementing WHO feeding guidelines for inpatient management of malnourished children. Journal of the College of Physicians and Surgeons--Pakistan: JCPSP. 2014;24(7):493-7.

41. Sadler K, Puett C, Mothabbir G, Myatt M. Community case management of severe acute malnutrition in southern Bangladesh. Boston: Tufts University; 2011.

42. Defourny I, Drouhin E, Terzian M, Taty M, Sekkenes J, Tectonidis M. Scaling up the treatment of acute childhood malnutrition in Niger. Field Exchange. 2006.

43. Dent N. Outpatient management of acute malnutrition in a Kenyan urban slum context: caseloads and challenges. Malawi Med J. 2009;21:123-59.

44. Dale NM, Myatt M, Prudhon C, Briend A. Using mid-upper arm circumference to end treatment of severe acute malnutrition leads to higher weight gains in the most malnourished children. PLoS One. 2013; 8(2):e55404

45. De Maayer T, Saloojee H. Clinical outcomes of severe malnutrition in a high tuberculosis and HIV setting. Archives of disease in childhood. 2011: archdischild 205039.

\section{Publisher's Note}

Springer Nature remains neutral with regard to jurisdictional claims in published maps and institutional affiliations. 